\title{
Kinematical fit and channel separation in np experiment
}

\author{
A.V. Belyaev ${ }^{1 \star}$, A.P. Jerusalimov ${ }^{1}$, and Yu.A. Troyan ${ }^{1}$ \\ ${ }^{1}$ Joint Institute for Nuclear Research, 141980 Dubna, Russia
}

\begin{abstract}
The method of channels separation in particle-particle collisions is presented. The kinematical fit and weights for tracks combinations are used. The Kolmogorov criterion is applied to check agreement between experimental and theoretical $\chi^{2}$ distributions. The investigations were made for data of np experiment in LHEP (JINR, Dubna) and for pp collisions in GSI (Darmstadt, Germany).
\end{abstract}

\section{Introduction}

The channel separation in many experiments in particle physics is often processed using value of a missing mass. In this case

1) kinematical parameters of particles are defined with a low precision

2) its values violate conservation laws

3) kinematical parameters of a neutral particle became inaccessible.

All this reasons make difficult an estimation of cross section errors. The kinematical fit and the proposed weight method allow to separate reaction channels eliminating reasons mentioned above.

\section{Data and results}

The neutron channel [1] of the 1m-H2-bubble chamber of the LHEP [2] worked in 70-90 years. Parameters of neutron beam are: momentum $p_{n} \sim(1-5) \mathrm{GeV} / \mathrm{c}$ with relative error $\delta p_{n} \sim 2.5 \%$ and with angle resolution $\Delta_{n} \sim 0.5 \mathrm{mRad}$. Total $\sim 10^{6}$ events of the np collision were registered at stereo-photos using the bubble chamber.

Taking into account energy range of a np collision, one can get a list of hadrons, which may be produced in some channels:

$$
\begin{array}{lccccc}
\text { positive charged particles: } & p & d & \pi^{+} & K^{+} & \Sigma^{+} \\
\text {negative charges particles: } & \pi^{-} & K^{-} & \Sigma^{-} & \Xi^{-} & \\
\text {neutral particles: } & \pi^{0} & K^{0} & \mathrm{n} & \Lambda & \Sigma^{0}
\end{array}
$$

What is a problem? By some reasons data have no identification of particles, i.e. several hypotheses of a particle may correspond to every track which may be produced by a charged particle. Therefore a 3-prong np collision, for example, may be considered with a lot of combinations of track hypotheses like in the table 1.

\footnotetext{
^e-mail: belyaev.bljv@yandex.ru
} 
Table 1.

\begin{tabular}{|l|l|}
\hline Combination & Channel \\
\hline$p p \pi^{-}$ & $n p \rightarrow p p \pi^{-}$ \\
\hline$p p \pi^{-} \pi^{0}$ & $n p \rightarrow p p \pi^{-} \pi^{0}$ \\
\hline$p p K^{-} \pi^{0}$ & $n p \rightarrow p p K^{-} K^{0}$ \\
\hline$p \pi^{+} \pi^{-} n$ & $n p \rightarrow p \pi^{+} \pi^{-} n$ \\
\hline$\pi^{+} p \pi^{-} n$ & $n p \rightarrow p \pi^{+} \pi^{-} n$ \\
\hline$p \pi^{+} \Sigma^{-} K^{0}$ & $n p \rightarrow p \pi^{+} \Sigma^{-} K^{0}$ \\
\hline
\end{tabular}

It is necessary to select the true combination (channel) of a collision. Two instruments are represented here to solve this problem: 1) Kinematical fit; 2) "Weighting".

The kinematical fit [3] is the conditional minimization of the functional $\chi^{2}=\left(\mathbf{x}-\mathbf{x}^{\mathbf{m}}\right) \mathbf{G}^{-1}\left(\mathbf{x}-\mathbf{x}^{\mathbf{m}}\right)$ with $\mathrm{r}$ constraint equations $F_{r}(x)=0 \quad(\mathrm{r}=1,2, \ldots)$ - number of degrees of freedom for $\left.\chi^{2}\right)$,

where $\mathbf{x}^{\mathbf{m}}$ - vector of measured kinematical parameters (conservation laws may be violated);

$\mathbf{G}^{-\mathbf{1}}$ - error matrix for $\mathbf{x}^{\mathbf{m}}$;

$\mathbf{x}$ - resulting vector of fitted kinematical parameters (conservation laws have no violations).

Constraint equations are usually based on conservation laws. Masses of secondary particles must be determined; they are used in constraint equations. Events which have only charged particles $(r=4)$ or (additionally) with a single neutral particle $(\mathrm{r}=1)$ may be processed using the kinematical fit.

If the conditional minimization process has a success, the kinematical fit:

- fits a set of more precise kinematical parameters which keeps following conservation laws;

- gives less errors for fitted kinematical parameters;

- calculates kinematical parameters (with its errors) for unmeasured neutral particle (in the case of a channel with a single neutral, i.e. unmeasured, particle);

- gives the value of the $\chi^{2}$ in the point of conditional minimum; the $\chi^{2}$ determines a confidence probability for a channel of an interaction been represented in the $\mathbf{x}^{\mathbf{m}}$.

If input data have no systematical errors then the experimental $\chi^{2}$ distribution must be a standard one. This theoretical approach was tested and confirmed (new) with the Kolmogorov's criterion. For this purpose:

1) a set of ideal events (a model of phase volume for any np collision) was generated with the Monte Carlo generator;

2) ideal kinematical parameters were smeared using random gauss errors; 3) such model data were fitted with the kinematical fit procedure.

Then the Kolmogorov's criterion test demonstrated that model $\chi^{2}$ distributions are in a fine agreement with standard ones. Conclusion (new): the kinematical fit procedure does not add systematical errors to resulting kinematical parameters (vector $\mathbf{x}$ above ).

Note that an agreement with some fixed confidence level, obtained due to Kolmogorov's criterion, is a necessary condition of absence of systematical errors in resulting kinematical parameters.

But the kinematical fit can reach a conditional minimum for several various track combinations in the same event. Here an attempt to solve this problem with the "weighting" is presented.

It turned out that the conditional minimum in fitting process was reached only for 3 combinations. These combinations were supplied with the corresponding $\chi^{2}$ :

$\begin{array}{ccccccc}1 & (7) & K^{-} & \mathrm{p} & K^{+} & \mathrm{n} & \chi_{1}^{2}=2.45 \\ 2 & (15) & \Sigma^{-} & \mathrm{p} & \pi^{+} & K^{0} & \chi_{2}^{2}=0.05 \\ 3 & (16) & \Sigma^{-} & \mathrm{p} & K^{+} & & \chi_{3}^{2}=3.11\end{array}$


The weight of a combination is proposed in the following way (new approach)

1) Confidence probabilities $\mathbf{H}_{\mathbf{j}}\left(\chi_{\mathrm{j}}^{2}, \mathrm{r}\right)=\int_{\chi_{\mathrm{j}}^{2}}^{\infty} \mathrm{p}(\mathrm{r}, \mathrm{z}) \mathrm{dz}, \quad(\mathrm{j}=1,2,3) \quad$, where $p(r, z)=\frac{z^{r / 2-1} \cdot e^{-z / 2}}{2^{r / 2} \times \Gamma(r / 2)}$ were calculated (figure 1).

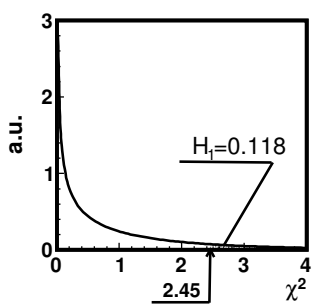

$K^{-} p K^{+} n \quad(r=1)$

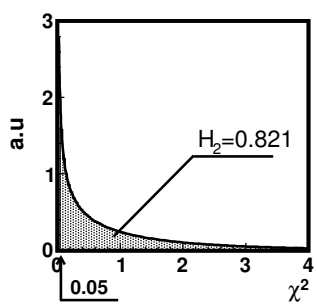

$\Sigma^{-} p \pi^{+} K^{0} \quad(r=1)$

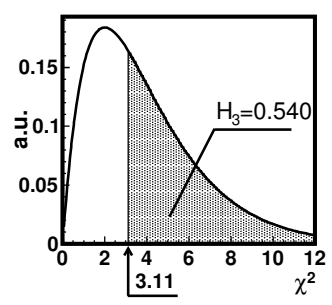

$\Sigma^{-} p K^{+}(r=4)$

Figure 1. On the calculation of confidence probabilities of combinations.

2) the weight of $j-t h$ combination is then defined as $w_{j}=\frac{H_{j}}{\sum_{i=1}^{3} H_{i}}, \quad(j=\overline{1,3})$

Results are combined in the table 2.

Table 2.

\begin{tabular}{|l|c|c|c|c|c|}
\hline Combination & $\mathrm{r}$ & $\chi^{2}$ & $H\left(\chi_{j}^{2}, r\right)$ & $\mathrm{w}$ & Channel \\
\hline$K^{-} p K^{+}$ & 1 & 2.45 & 0.118 & 0.080 & $p K^{+} K^{-} n$ \\
\hline$\Sigma^{-} p \pi^{+} K^{0}$ & 1 & 0.005 & 0.821 & 0.555 & $p \pi^{+} \Sigma^{-} K^{0}$ \\
\hline$\Sigma^{-} p K^{+}$ & 4 & 3.11 & 0.540 & 0.365 & $p K^{+} \Sigma^{-}$ \\
\hline
\end{tabular}

The "weight-method" differs from the method of "preference rules" which was used earlier. Using preference rules one selects only sole combination from the ones mentioned in example above. So, under the preference rules 1 ) combination with $r=4$ has preference before all others with $r=1 ; 2$ ) combination with minimal $\chi^{2}$ has preference over all others (with the same value of $r$ ).

In the case of using the described weight-method the Kolmogorov's criterion produces an agreement (between experimental and standard $\chi^{2}$ distributions), which is better than with some other methods that we used. This fact we can demonstrate with values of argument $\lambda$ of the Kolmogorov's function $K(\lambda)$. In accordance with the Kolmogorov's criterion - smaller values of its argument $\lambda$ correspond to bigger values of confidence probability $1-K(\lambda)$, where the Kolmogorov's function is $K(\lambda)=$ $\sum_{k=-\infty}^{\infty}(-1)^{k} e^{2 k^{2} \lambda^{2}}$. Figures 2 and 3 shows differences between results of the preference rules and the weight-method. One can see that values of $\lambda$ is much smaller for the weight-method.

Channel separation for all data was realized using the kinematical fit procedure and the described weight-method. Results of channel separation are given in tables 3 - 7 .

The column " $\sum(w)$ " contains sums of weights for all minimized combinations of given channel. It should be considered as effective number of events, corresponding to a given channel.

In cases when $\sum(w)>1000$ the Kolmogorov's $\lambda$ are shown.

One can see that the rounded value " $\sum \sum(w)$ " equal to number of all events which have at least one minimized combination. 

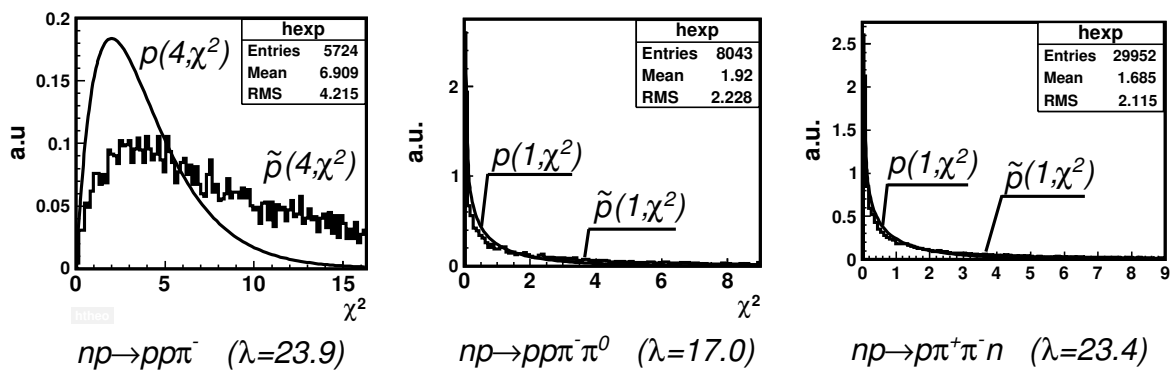

Figure 2. The preference rules: experimental functions $\tilde{p}\left(r, \chi^{2}\right)$ combined with theoretical ones $p\left(r, \chi^{2}\right)$.
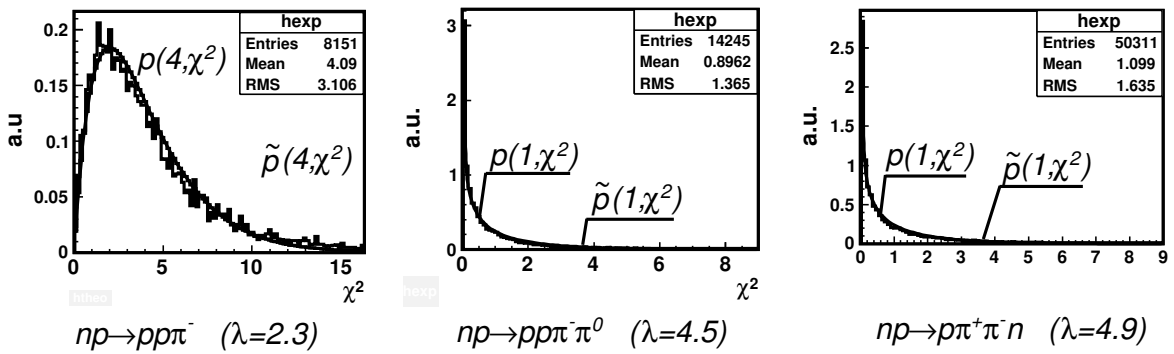

Figure 3. The weight method: experimental functions $\tilde{p}\left(r, \chi^{2}\right)$ combined with theoretical ones $p\left(r, \chi^{2}\right)$.

The contribution for a channel is calculated as $\left(\sum(w) /(\right.$ total events $\left.)\right) * 100$.

For some channels of 1- and 3-prong events it was possible to compare branching ratios with ones published in [4] where they were calculated using preference rules and for smaller set of channels. Data shown in tables 3 - 7 should be considered as preliminary due to absence of the particle identification in events what makes values of the Kolmogorov's $\lambda$ in some channels too big.

\section{Conclusion}

1. The method of channels separation in particle-particle collisions using the kinematical fit and weights for track combinations is presented.

2. The Kolmogorov's criterion was applied to check agreement between experimental and theoretical $\chi^{2}$ distributions.

3. Channel separation using weights for 1-, 3- and 7-prong events of np collision (for various values of $p_{n}$ ) was realised.

4. The procedure of Kinematical Fit allowed to refine the parameters of the registered particles and to reconstruct the parameters of the neutral ones.

\section{References}

[1] Yu.D. Beznogikh et al., Prib.Tekh.Eksp. 4, 202 (1969) (in Russian)

[2] V.V. Glagolev, http://he.jinr.ru/1mHBC/index.html

[3] J.P. Berge, F.T. Solmitz and H.D.Taft. Rev.Sci.instrum., 32, 538-548 (1961)

[4] C. Besliu et al., Yad.Fiz. 43, 888-892 (1986) 
Table 3. 3-prong events at $P_{n}=5.20 \mathrm{GeV} / \mathrm{c}$

\begin{tabular}{|l|c|c|c|}
\hline Channel & $\Sigma(w)$ & Kolmogorov's $\lambda$ & Contribution (\%) \\
\hline$d \pi^{+} K^{-} K^{0}$ & 1036.84 & 1.6 & $1.5 \pm 0.1$ \\
\hline$p K^{+} K^{-} n$ & 2271.32 & 5.4 & $3.2 \pm 0.1$ \\
\hline$d \pi^{+} \pi^{-} \pi^{0}$ & 3186.41 & 1.7 & $4.5 \pm 0.1$ \\
\hline$p K^{+} \pi^{-} \Sigma^{0}$ & 3697.29 & 6.5 & $5.2 \pm 0.1$ \\
\hline$p K^{+} \pi^{-} \Lambda$ & 4739.72 & 9.0 & $6.7 \pm 0.1$ \\
\hline$p p \pi^{-} \pi^{0}$ & 6018.38 & 4.5 & $8.5 \pm 0.1$ \\
\hline$p p \pi^{-}$ & 7018.73 & 2.3 & $9.9 \pm 0.1$ \\
\hline$p \pi^{+} \pi^{-} n$ & 30695.38 & 4.9 & $43.1 \pm 0.2$ \\
\hline$\Sigma(\Sigma(w))$ & 60140.97 & & $84.7 \pm 0.3$ \\
\hline Events with fit (M) & 60141 & & 84.7 \\
\hline Events with no fit (K) & 10882 & & 15.3 \\
\hline Total events (N) & 71023 & & 100.0 \\
\hline
\end{tabular}

Table 4. 1-prong events at $P_{n}=5.20 \mathrm{GeV} / \mathrm{c}$

\begin{tabular}{|c|c|c|c|}
\hline Channel & $\Sigma(w)$ & Kolmogorov's $\lambda$ & Contribution (\%) \\
\hline$d \pi^{0}$ & 311.77 & & $2.9 \pm 0.2$ \\
\hline$p n$ & 4465.24 & 1.9 & $42.2 \pm 0.6$ \\
\hline$\Sigma(\Sigma(w))$ & 4777.00 & & $45.1 \pm 0.7$ \\
\hline Events with fit (K) & 4777 & & 45.1 \\
\hline Events with no fit (M) & 5812 & & 54.9 \\
\hline Total events (N) & 10589 & & 100.0 \\
\hline
\end{tabular}

Table 5. 1-prong events at $P_{n}=2.23 \mathrm{GeV} / \mathrm{c}$

\begin{tabular}{|c|c|c|c|}
\hline Channel & $\Sigma(w)$ & Kolmogorov's $\lambda$ & Contribution (\%) \\
\hline$d \pi^{0}$ & 443.48 & & $3.8 \pm 0.2$ \\
\hline$p n$ & 5476.57 & 2.4 & $47.3 \pm 0.6$ \\
\hline$\Sigma(\Sigma(w))$ & 5920.00 & & $51.1 \pm 0.7$ \\
\hline Events with fit (K) & 5920 & & 51.1 \\
\hline Events with no fit (M) & 5665 & & 48.9 \\
\hline Total events (N) & 11585 & & 100.0 \\
\hline
\end{tabular}


Table 6. 1-prong events at $P_{n}=1.43 \mathrm{GeV} / \mathrm{c}$

\begin{tabular}{|c|c|c|c|}
\hline Channel & $\Sigma(w)$ & Kolmogorov's $\lambda$ & Contribution (\%) \\
\hline$d \pi^{0}$ & 411.72 & & $6.7 \pm 0.3$ \\
\hline$p n$ & 3995.28 & 1.5 & $64.6 \pm 1.0$ \\
\hline$\Sigma(\Sigma(w))$ & 4407.00 & & $71.3 \pm 1.1$ \\
\hline Events with fit (K) & 4407 & & 71.3 \\
\hline Events with no fit $(\mathrm{M})$ & 1775 & & 28.7 \\
\hline Total events (N) & 6128 & & 100.0 \\
\hline
\end{tabular}

Table 7. 7-prong events at $P_{n}=5.20 \mathrm{GeV} / \mathrm{c}$

\begin{tabular}{|l|c|c|}
\hline Channel & $\Sigma(w)$ & Contribution (\%) \\
\hline$d \pi^{+} \pi^{+} \pi^{+} \pi^{-} \pi^{-} \pi^{-}$ & 2.55 & $<1.0$ \\
\hline$d \pi^{+} \pi^{+} \pi^{+} \pi^{-} \pi^{-} \pi^{-} \pi^{0}$ & 7.07 & $2.0 \pm 0.8$ \\
\hline$p \pi^{+} \pi^{+} \pi^{+} \pi^{-} \pi^{-} \pi^{-} n$ & 40.17 & $11.4 \pm 1.8$ \\
\hline$p p \pi^{+} \pi^{+} \pi^{-} \pi^{-} \pi^{-} \pi^{0}$ & 48.44 & $13.7 \pm 2.0$ \\
\hline$p p \pi^{+} \pi^{+} \pi^{-} \pi^{-} \pi^{-}$ & 189.78 & $53.8 \pm 3.9$ \\
\hline$\Sigma(\Sigma(w))$ & 288.00 & $81.6 \pm 4.8$ \\
\hline Events with fit (M) & 288 & 81.6 \\
\hline Events with no fit (K) & 65 & 18.4 \\
\hline Total events (N) & 353 & 100.0 \\
\hline
\end{tabular}

